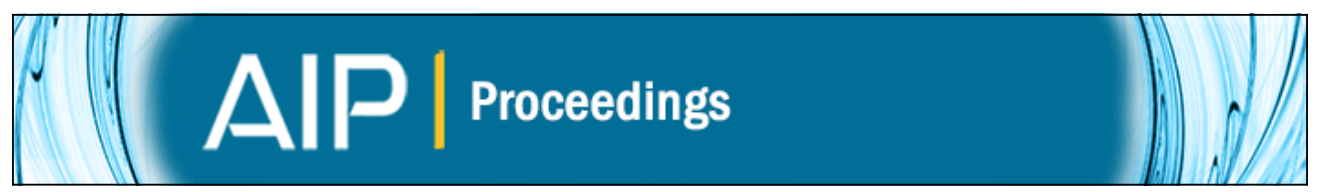

\title{
Attraction and repulsion of multi-color laser beams in plasmas: a computational study
}

\author{
S. A. Yi, S. Kalmykov, and G. Shvets
}

Citation: AIP Conference Proceedings 1086, 303 (2009); doi: 10.1063/1.3080923

View online: http://dx.doi.org/10.1063/1.3080923

View Table of Contents:

http://scitation.aip.org/content/aip/proceeding/aipcp/1086?ver=pdfcov

Published by the AIP Publishing

\section{Articles you may be interested in}

Experimental and computational study of complex shockwave dynamics in laser ablation plumes in argon atmosphere

Phys. Plasmas 19, 083504 (2012); 10.1063/1.4745867

Plasma jets produced in a single laser beam interaction with a planar target

Phys. Plasmas 13, 062701 (2006); 10.1063/1.2206171

Ion Coulomb explosion of clusters by a Gaussian laser beam

Phys. Plasmas 10, 4085 (2003); 10.1063/1.1608017

Neutral Beam Generation by Laser Irradiation of Thin Foils

AIP Conf. Proc. 634, 329 (2002); 10.1063/1.1514307

Overview of laser particle acceleration in an underdense plasmas

AIP Conf. Proc. 611, 303 (2002); 10.1063/1.1470316 


\title{
Attraction and repulsion of multi-color laser beams in plasmas: a computational study
}

\author{
S. A. Yi, S. Kalmykov and G. Shvets \\ Department of Physics and Institute for Fusion Studies, The University of Texas at Austin, One \\ University Station C1500, Austin, Texas 78712
}

\begin{abstract}
The nonlinear interaction of high-power multi-color laser beams in plasmas is investigated numerically. Both the relativistic mass increase and the driven plasma wave contribute to the mutual beam-beam interaction and to the development of the electromagnetic cascade. The propagation of the individual cascade sidebands is modelled in the paraxial approximation. The resulting set of coupled nonlinear envelope equations is solved numerically using a newly developed pseudospectral method. We predict that two beams intersecting in the plasma can either attract or deflect each other depending on whether their frequency detuning is slightly below or above the electron Langmuir frequency.
\end{abstract}

Keywords: multi-color laser beams in plasmas, electromagnetic cascading

PACS: 52.35.Mw, 52.38.-r, 52.38.Hb

\section{INTRODUCTION}

Long-distance propagation of laser beams through plasmas is of great importance to laser-plasma accelerators because it affects the total single-stage energy gain of the accelerated electrons. While even the nonlinear propagation of a single laser pulse can be fairly complex due to the phenomenon of relativistic self-focusing $[1,2,3]$, multiple beam propagation reveals a range of even more complicated nonlinear effects. Laser beams can interact with each other through the relativistic mass effect $[4,5,6,7]$, as well as through the electron density perturbation (electron plasma wave, EPW) driven by the ponderomotive force of the lasers $[5,6,7,8]$. In the collinear geometry, the latter interaction is significant because the relativistic self-focusing may be enhanced or suppressed by the cross-focusing or defocusing effect of the EPW, depending on the detuning frequency $[5,7]$. In the non-collinear geometry, same-frequency beams can either attract or repel each other depending on their relative phase [6]. This effect is due to the relativistic and ponderomotive nonlinearities.

In this Report, we explore the nonlinear interaction between laser beams of different colors; their difference frequency $\Omega$ can be close, but not equal, to the electron Langmuir frequency $\omega_{p}=\left(4 \pi e^{2} n_{0} / m_{e}\right)^{1 / 2}$ (where $n_{0},-e$, and $m_{e}$ are, respectively, the electron background density, charge, and rest mass). First, we present a paraxial model for the propagation of multi-color laser beams in plasmas. Next, we propose a numerical method for solving the nonlinear paraxial equations of the model. We then present preliminary numerical results which demonstrate mutual attraction and repulsion between two frequency-detuned beams in plasmas, and conclude with an outline for future work. 


\section{THEORETICAL MODEL}

We present here a theoretical model for the propagation, electromagnetic cascading, and mutual interaction in rarefied plasmas of weakly-relativistic frequency-detuned laser beams. The laser propagation is modelled in the paraxial approximation, taking into account the group velocity dispersion, and beam-beam interaction through both the density perturbations due to the plasma wake and the relativistic mass corrections. Long laser beams detuned in frequency by $\Omega \neq \omega_{p}$ are assumed, so that the density perturbations are due to the instantaneous (on the scale of the laser pulse duration) plasma response to the ponderomotive beat wave. This is a $3 \mathrm{D}$ model, building upon an earlier 1D model [9] for the electromagnetic cascading of multi-color laser beams in plasmas.

Consider two laser beams shifted in frequency by $\Omega \ll \omega_{0}$ focused onto a plasma boundary at $z=0$, where $z$ is the propagation variable, and $\omega_{0}$ is the fundamental frequency of beam " 0 ". This bi-color laser beam can be described by the vector potential (normalized to $\left.m_{e} c^{2} /|e|\right) \mathbf{a}\left(\mathbf{x}_{\perp}, z=0, \xi\right)=\operatorname{Re}\left[\mathbf{e}_{0} a\left(\mathbf{x}_{\perp}, z=0, \xi\right)\right]$, where

$$
a\left(\mathbf{x}_{\perp}, 0, \xi\right)=e^{-i k_{0} \xi}\left[a_{0}\left(\mathbf{x}_{\perp}, 0, \xi\right)+a_{1}\left(\mathbf{x}_{\perp}, 0, \xi\right) e^{-i k_{\Omega} \xi}\right] .
$$

Here, $\mathbf{e}_{0}$ is the unit complex polarization vector, $\mathbf{x}_{\perp}=(x, y), \xi=c t-z, k_{0}=\omega_{0} / c$, and $k_{\Omega}=\Omega / c$. As the beams propagate through the plasma, their ponderomotive force produces a co-moving modulation of refractive index; the latter, in turn, produces new sidebands in the laser spectrum,

$$
a\left(\mathbf{x}_{\perp}, z>0, \xi\right)=\sum_{l=-\infty}^{+\infty} a_{l}\left(\mathbf{x}_{\perp}, z>0, \xi\right) e^{-i k_{l} \xi},
$$

where $k_{l}=k_{0}+l k_{\Omega}, l$ is an integer. The evolution of the cascade envelopes is governed by the set of coupled nonlinear paraxial equations:

$$
\left(2 i \partial / \partial z+\nabla_{\perp}^{2}\right) a_{l}=k_{l}^{2}\left(1-\eta_{l}^{2}\right) a_{l},
$$

where $\eta_{l}$ are the nonlinear refractive indices given by

$$
\eta_{l}^{2}=1-\frac{k_{p}^{2}}{k_{l}^{2}}\left[\left(l \frac{\Omega}{\omega_{0}}\right)^{2}+\frac{1}{2} \sum_{m} \frac{a_{m+l}}{a_{l}}\left(N_{m}-\frac{\rho_{m}}{2}\right)\right] .
$$

Here, $k_{p}=\omega_{p} / c$, the first term in the square brackets represents the group velocity dispersion, and the second term represents the nonlinear response of the plasma, i.e., the electron density perturbations and the relativistic mass increase of plasma electrons. $\rho_{l}=\sum_{m} a_{m} a_{m+l}^{*}$ are the laser intensity moments, and $N_{l}=\delta n_{l} / n_{0}$ are the normalized harmonics of the driven electron density perturbations derived from the equations of cold electron hydrodynamics:

$$
N_{l}\left(\mathbf{x}_{\perp}, z, \xi\right) \approx \frac{1}{2} \frac{(l \Omega)^{2}}{(l \Omega)^{2}-\omega_{p}^{2}} \rho_{l}\left(\mathbf{x}_{\perp}, z, \xi\right),
$$


TABLE 1. A comparison of the efficiency of numerical methods. Numerical parameters: $d x=d y=.1562 r_{00}, N_{x}=N_{y}=128$.

\begin{tabular}{lrrr}
\hline & $\mathbf{d z} / \mathbf{z}_{\mathbf{R}}$ & $\mathbf{z}$-steps & runtime (s) \\
\hline Split-step Fourier (2nd order) & 0.005 & 200 & 306 \\
Split-step Fourier (4th order) & 0.025 & 40 & 192 \\
Pseudospectral & 0.01 & 100 & 102 \\
\hline
\end{tabular}

where $n_{e}\left(\mathbf{x}_{\perp}, z, \xi\right)-n_{0}=\frac{1}{2} \sum_{l} \delta n_{l}\left(\mathbf{x}_{\perp}, z, \xi\right) e^{i l k_{\Omega} \xi}$. Equation (5) assumes an instantaneous plasma response to the periodic ponderomotive force (plasma does not have a memory), which is justified when $\left|\Omega-\omega_{p}\right| \tau_{L}>1$, where $\tau_{L}$ is the laser beam length [10]. The electron density perturbations are assumed to be phase-locked with the laser ponderomotive force, which is justified for the non-resonant beat wave $[9,10]\left|\Omega-\omega_{p}\right| \gg 3 \omega_{R L}$, where $\omega_{R L}=(1 / 4)\left(3\left|a_{0} a_{1}\right|^{2} / 2\right)^{1 / 3}$ is the Rosenbluth-Liu saturation frequency [11].

\section{NUMERICAL METHODS FOR SOLVING EQ. 3}

Although the theoretical model described above is fully $3 \mathrm{D}$, we reduce the physical dimension to 2D, assuming infinitely long laser beams. Thus, we solve the system of coupled nonlinear paraxial equations (3) numerically in a transverse subspace, using a pseudospectral method which calculates the spacial dispersion term in Fourier space, and the nonlinear plasma response in physical space. Taking the Fourier transform of equations (3) and rewriting,

$$
2 i \partial \tilde{a}_{l} / \partial z=\left(k_{x}^{2}+k_{y}^{2}\right) \tilde{a}_{l}+k_{l}^{2} \mathscr{F}\left[\left(1-\eta_{l}^{2}\right) a_{l}\right],
$$

where the tildes and $\mathscr{F}$ denote Fourier transformed quantities, and $k_{x}, k_{y}$ are the $x, y$ wavenumbers. The set of coupled ordinary differential equations (6) can be integrated in Fourier space using a fourth-order Runge-Kutta method. Since the nonlinear part $\left(1-\eta_{l}^{2}\right) a_{l}$ must first be evaluated in physical space before taking its Fourier transform, each full fourth-order Runge-Kutta step in $z$ requires taking 4 fast Fourier transforms (FFT), and 4 inverse FFTs.

We have found that this pseudospectral numerical scheme for solving the nonlinear wave equations (3) is comparable in efficiency to a fourth-order version of the wellknown Split-Step Fourier method $[12,13]$. In table 1, we show sample runtimes for some typical physical and numerical parameters, where for each numerical method we propagate the laser beam up to $z=z_{R}$, and take the largest steps in $z$ possible while still conserving energy to a factor $10^{-4}$. The pseudospectral method has the shortest runtime for a given accuracy.

\section{MUTUAL INTERACTION BETWEEN NON-COLLINEAR BEAMS}

Consider two laser beams shifted in frequency by $\Omega \ll \omega_{0}$ propagating non-collinearly in a plasma. If these beams are close enough to interact, the beat-wave driven electron 
(a)

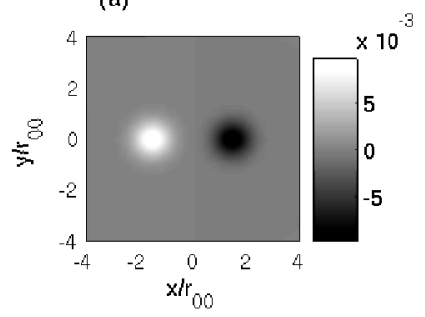

(c)

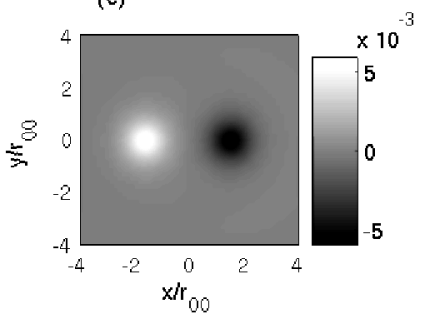

(b)

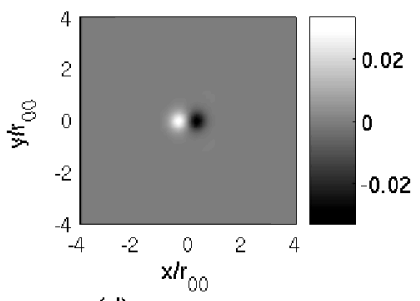

(d)

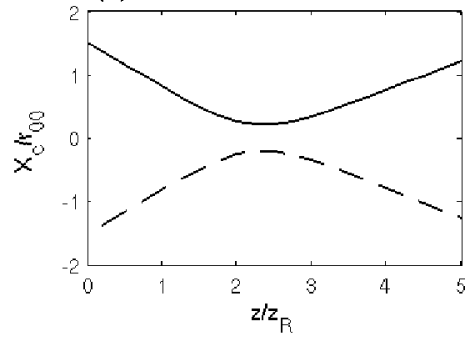

FIGURE 1. Mutual interaction between non-colinear beams detuned by $\Omega=1.25 \omega_{p}$. Individual laser beam intensities (conveniently approximated by $\left|a_{0}\right|^{2}-\left|a_{1}\right|^{2}$ for better visualization) are plotted at (a) $z=0$, (b) $z=2.5 z_{R}$, and (c) $z=5.0 z_{R}$. (d) Centroid positions of the higher frequency beam (solid line) and the lower frequency beam (dashed line).

density perturbation acts as a co-moving periodic channel that is focusing when $\Omega<$ $\omega_{p}$ and defocusing when $\Omega>\omega_{p}$ [7]. Thus, the two laser beams mutually attract if the difference frequency is slightly below the plasma frequency, and repel if the difference frequency is slightly above. This effect is of a different nature than the earlier described [6] mutual interaction between same-color beams.

Simulation results for two colliding laser beams (labelled $l=0,1$ ) are shown in Fig. 1. The beams initially have axi-symmetric Gaussian intensity profiles in the cross-sections perpendicular to their propagation axes. At $z=0$, they are separated in the $x$ direction by 3 beam radii and aimed towards each other at incident angles $\theta_{0}= \pm 0.014^{\circ}$ to the $z$ axis. The difference frequency is $\Omega=1.25 \omega_{p}$, and each beam has power $P_{0}=P_{1}=0.95 P_{c r}$, where $P_{c r}=16.2\left(\omega_{0} / \omega_{p}\right)^{2} \mathrm{GW}$ is the critical power for relativistic self-focusing [3]. For weakly-relativistic beams with $a_{00}=a_{01}=0.1$, the initial radii of the beams are $k_{p} r_{00}=k_{p} r_{01}=55$. The background plasma density is $n_{0}=1.7 \times 10^{17} \mathrm{~cm}^{-3}$, and $\lambda_{0}=0.8 \mu \mathrm{m}$ corresponds to $\omega_{p} / \omega_{0}=0.01$.

Figures 1(a), 1(b), and 1(c) show the intensities of the individual beams at $z=0$, $z=2.5 z_{R}$, and $z=5.0 z_{R}$, respectively, where $z_{R}=k_{0} r_{00}^{2} / 2$ is the Rayleigh length. Beam centroid dynamics in the $x-z$ plane are displayed in Fig. 1(d), where $\mathbf{X}_{c}=$ $\int d \mathbf{x}_{\perp} \mathbf{x}_{\perp}|a|^{2} / \int d \mathbf{x}_{\perp}|a|^{2}$. Figure 1 clearly confirms that the two beams have scattered off of each other due to the repulsive mutual interaction.

To emphasize the importance of the frequency detuning between the beams, we have simulated the interaction between two laser beams with the same parameters, except 
(a)

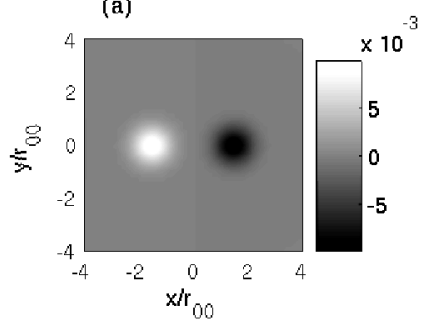

(c)

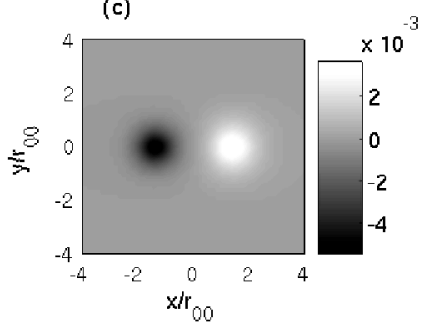

(b)
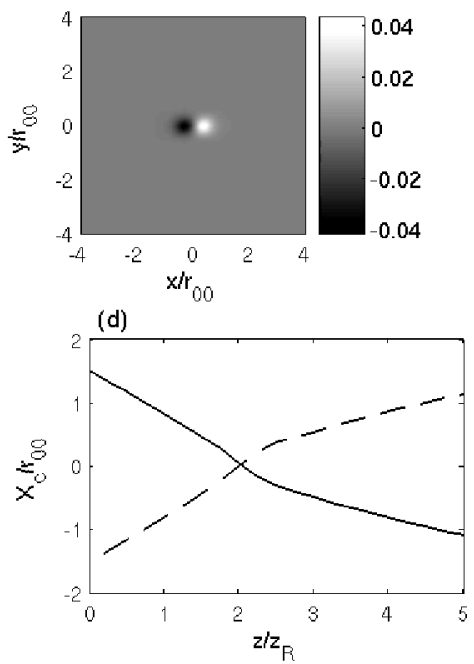

FIGURE 2. Same as in Fig. 1, except that the beams are strongly detuned by $\Omega=3.25 \omega_{p}$.

that $\Omega=3.25 \omega_{p}$. It is apparent from Fig. 2 that the two beams have passed through each other, in contrast with Fig. 1. It is clear from Figs. 1 and 2 that the difference frequency is an important parameter in the mutual interaction between multi-color laser beams. The nonlinear susceptibility $\chi_{l}=\left(k_{l}^{2} / k_{p}^{2}\right)\left(1-\eta_{l}^{2}\right)$ for both cases at $z=2.0 z_{R}$ is shown in Fig. 3. In Fig. 3, the darker regions correspond to higher refractive index $\eta_{l}$, to which the laser beams are drawn. We further note that these simulations are a work in progress, and thus the effect of electromagnetic cascading has been neglected, taking into account only the two-beam interactions. Understanding the effects of sideband generation on the beam-beam interaction is the subject of future work.

\section{SUMMARY AND FUTURE WORK}

In summary, we have presented a reduced 3D model for the propagation and electromagnetic cascading of high-power (close to the relativistic self-focusing threshold) multicolor laser beams in rarefied plasmas. Assuming long laser pulses, we have numerically implemented our model to investigate the mutual interaction between two almost copropagating frequency-detuned laser beams aimed towards each other. The defocusing effect of the beat wave driven density perturbations has been shown to be significant in the mutual attraction or repulsion between the beams.

In the forthcoming publications, we will investigate the effect of the growth of electromagnetic cascade sidebands on this mutual interaction. The effects of the finite pulse duration and the resulting non-stationary plasma response will also be investigated. 


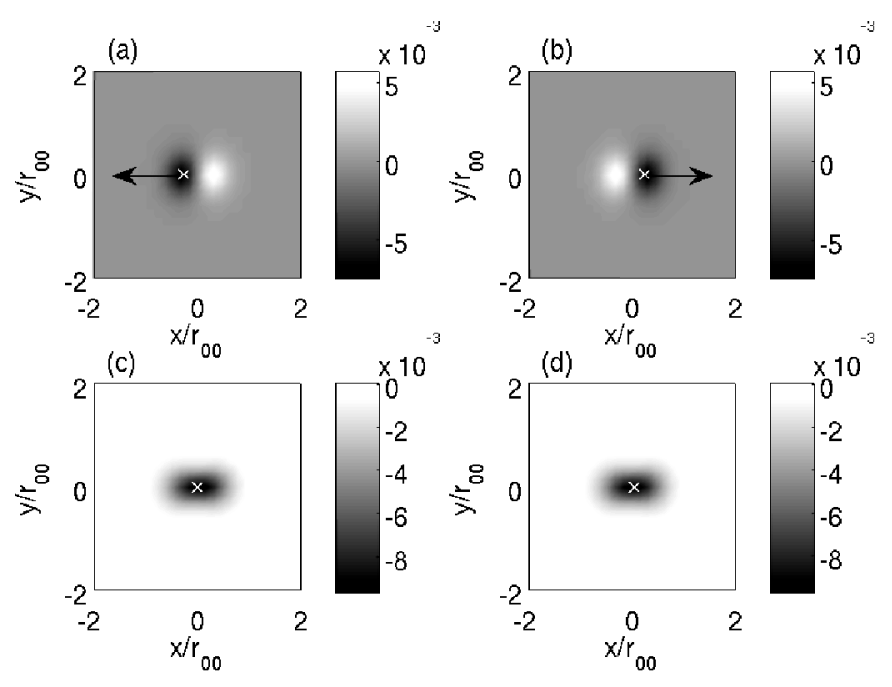

FIGURE 3. Nonlinear susceptibility $\chi_{l}=\left(k_{l}^{2} / k_{p}^{2}\right)\left(1-\eta_{l}^{2}\right)$ at $z=2.0 z_{R}$. (a), (b) $l=0,1$ for $\Omega=1.25 \omega_{p}$, and (c), (d) $l=0,1$ for $\Omega=3.25 \omega_{p}$. Centroids are marked with $x$ 's. Darker regions correspond to higher refractive index $\eta_{l}$ in (a) and (b). Arrows show direction of effective force on centroids. Here $l=0$ corresponds to lower frequency $\omega=\omega_{0}$, and $l=1$ corresponds to higher frequency $\omega=\omega_{0}+\Omega$.

\section{ACKNOWLEDGMENTS}

This work is supported by the U.S. Department of Energy grants No. DE-FG0204ER54763, DE-FG02-04ER41321, DE-FG02-07ER54945, and by the NSF grant PHY0114336 administered by the FOCUS Center at the University of Michigan, Ann Arbor.

\section{REFERENCES}

1. A. G. Litvak, Sov. Phys.-JETP 30, 344 (1970).

2. C. E. Max, J. Arons and A. B. Langdon, Phys. Rev. Lett. 33, 209 (1974).

3. G. Z. Sun, E. Ott, Y. C. Lee and P. Guzdar, Phys. Fluids 30, 526 (1987).

4. E. Esarey, A. Ting, and P. Sprangle, Appl. Phys. Lett. 53, 1266 (1988).

5. P. Gibbon, Phys. Fluids B 2, 2196 (1990).

6. C. Ren et al., Phys. Plasmas 9, 2354 (2002).

7. S. Kalmykov, S. A. Yi and G. Shvets, All-optical control of nonlinear self-focusing of laser beams in plasma beat wave accelerators (to appear in Plasma Phys. Control. Fusion).

8. P. Gibbon and A. R. Bell, Phys. Rev. Lett. 61, 1599 (1988); 61, 2509 (1988); 65, 1962 (1990); E. Esarey and A. Ting, ibid., 65, 1961 (1990).

9. S. Kalmykov and G. Shvets, Phys. Plasmas 13, 056707 (2006).

10. S. Kalmykov and G. Shvets, Phys. Rev. E. 73, 046403 (2006).

11. M. N. Rosenbluth and C. S. Liu, Phys. Rev. Lett. 29, 701 (1972).

12. T. R. Taha and M. J. Ablowitz, J. Comp. Phys. 55, 203 (1984).

13. G. M. Muslu and H. A. Erbay, Math. Comp. Sim. 67, 581 (2005). 